\title{
sciendo
}

\author{
ŁUKASZ KWIETNIEWSKI ${ }^{1}$, ANGELIKA KUCZYŃSKA ${ }^{1}$, WIKTOR KUPISZ" \\ MAGDALENA KWIETNIEWSKA ${ }^{2}$, WITOLD KRUPSKI ${ }^{1}$
}

\section{Clinical implications of breast ultrasound elastography}

\begin{abstract}
Breast cancer is the most common cancer in Polish women and the second leading cause of cancer deaths. Screening mammography is the only recognized method for early detection of malignant neoplasm and provides to reduction in cancer related deaths so far. The sensitivity of this examination is strongly influenced by the structure of the breast and is definitely lower for breasts of type 2 and 3 in accordance to American College of Radiology. That is why it is suggested that women younger than 35 years should be examined with ultrasound if necessary, and for women 35 years and older it is recommended to perform mammography, as the primary breast study. Ultrasound elastography is noninvasive imaging technique which can contribute to increase the sensitivity and specificity of recognition breast lesions. There are two main types of elastography: static and shear wave one. According to tissue stiffness, static elastography encodes lesions using a 5-point color scale - the Tsukuba University Score. Improvements in the interpretation of non-invasive examinations with using ultrasound elastography were discussed. It is considered that addition ultrasound elastography to ultrasound B-mode can play an important role in verification focal lesions in the breast.
\end{abstract}

Keywords: elasticity imaging techniques, breast neoplasm, Tsukuba University Score.

DOI: $10.2478 /$ pjph-2020-0004

\section{INTRODUCTION}

\section{Epidemiology}

Breast cancer is the most common cancer in Polish women. Female breast cancer represents $22 \%$ of all new cancer cases in Poland. The number of malignant breast tumors has more than doubled in the last three decades. The highest increase in risk is observed among women aged 50-69 years. There is also a significant tendency to increase in incidence in adult women in premenopausal age (20-49 years). In this group of patients, the incidence rate of breast cancer was almost 1.7 times higher in the last three decades [1]. Breast cancer is the second leading cause of cancer deaths (approximately $15 \%$ of all cancer death) among women [1].

\section{Diagnostic methods}

Screening mammography is the only recognized method for early detection of breast cancer and is associated with a reduction in breast cancer deaths across a range of study designs [2]. The sensitivity of mammography (MMG) is strongly influenced by the structure of the breast. The American College of Radiology (ACR) described four categories of breast density: almost entirely fat ( $>75 \%$ fat density), catered fibroglandular densities $(51-75 \%$ fat density), heterogeneously dense $(51-75 \%$ glandular density), extremely dense $(>75 \%$ glandular density) [3]. The sensitivity of MMG is definitely lower for breasts of type 2 and 3. The sensitivity of MMG in type 1 is $87 \%$, while in very dense breasts only $62.9 \%$.
In turn, the specificity of MMG in glandular breasts is $89.1 \%$, and in fatty breasts it reaches nearly $97 \%$ [4]. Therefore, it is suggested that women younger than 35 years should be examined with ultrasound if necessary, and for women 35 years and older is recommended to perform MMG, as the primary breast imaging modality [5]. It is necessary to search for new, available diagnostic methods that, by increasing the sensitivity and specificity of diagnostic tests, will contribute to a decrease in the percentage of false negative and false positive results. Ultrasound elastography is considered to be such a method.

\section{Ultrasound elastography}

Ultrasound elastography (USE) is noninvasive imaging technique that can be used in medicine to define stiffness of the tissues. USE was introduced in clinical practice in the 1990's [6]. This technique is highly sensitive to tissue structural changes resulting from specific pathological or physiological processes [7]. Combined with conventional imaging modalities, elastography can evaluate the stiffness of breast lesions and potentially can help differentiate benign tumor from malignant ones [8]. Recent studies have shown that the USE delivers higher image quality compared to conventional ultrasound and MMG in the diagnosis of breast cancer. Moreover, it contributes to increase in the specificity of the examination and thus reduces performing diagnostic breast biopsy [8]. USE may be divided into two main groups depending on the stimulation method and measurements: static elastography and shear wave elastography [9]. 
Static (compressive) elastography is a method of imaging the displacement of tissues subjected to an external pressure. This type of USE is based on compressing the examined lesion and assessing its deformation based on changes in ultrasonic echoes [10]. It is recommended pressure depth of 1-2 mm with a compressing frequency of 1-2 times per second [11]. The values of strain inversely correlate with tissue stiffness. The higher is hardness of the tissue, the lower is its deformation under compression, and thus the ultrasonographic images before and after pressure are similar. On the other hand, the soft tissues are more deformed under compression and thus pre- and post- compression significantly differ [10]. According to tissue stiffness, Itoh et al. proposed encoding tissue changes using a 5-point color scale - the Tsukuba University Score [11].

Tsukuba scale of breast neoplastic lesions divided them into five grades:

- Grade 1. Benign lesion. Full deformation is present in the entire hypoechoic area (Fig. 1).

- Grade 2. Benign lesion. Deformation is present in most of the hypoechoic area. A pattern typical of fibroadenomas (Fig. 2).

- Grade 3. Inability to explicitly exclude a malignant lesion. Deformation is present only on the edges of the lesion (Fig. 3).

- Grade 4. Suspected malignancy. Deformation is absent in the entire hypoechoic area (Fig. 4).

- Grade 5. High probability of a malignant lesion. Deformation is absent in the entire hypoechoic area of the lesion and the surrounding tissues (Fig. 5).

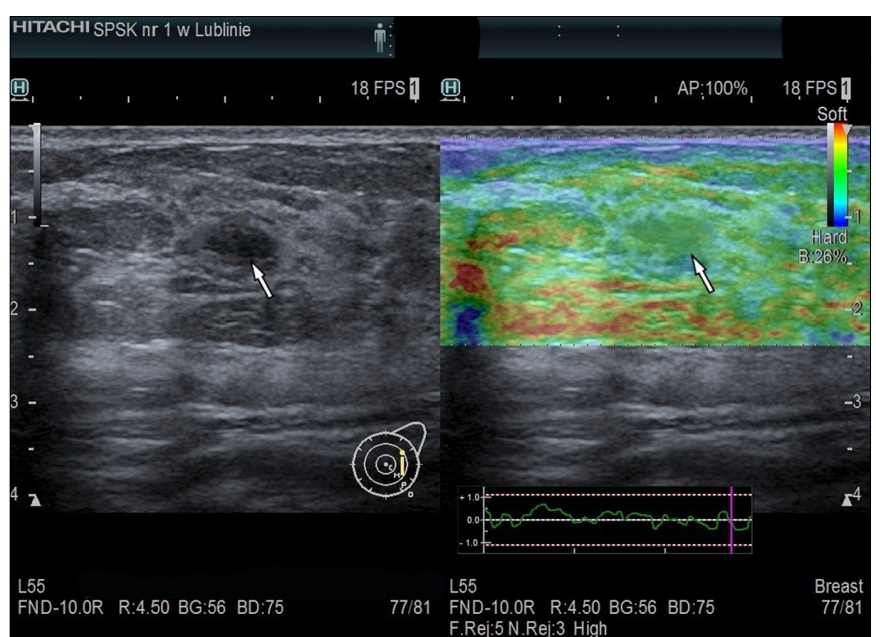

FIGURE 1. Hypoechoic lesion classified as Tsukuba score 1.

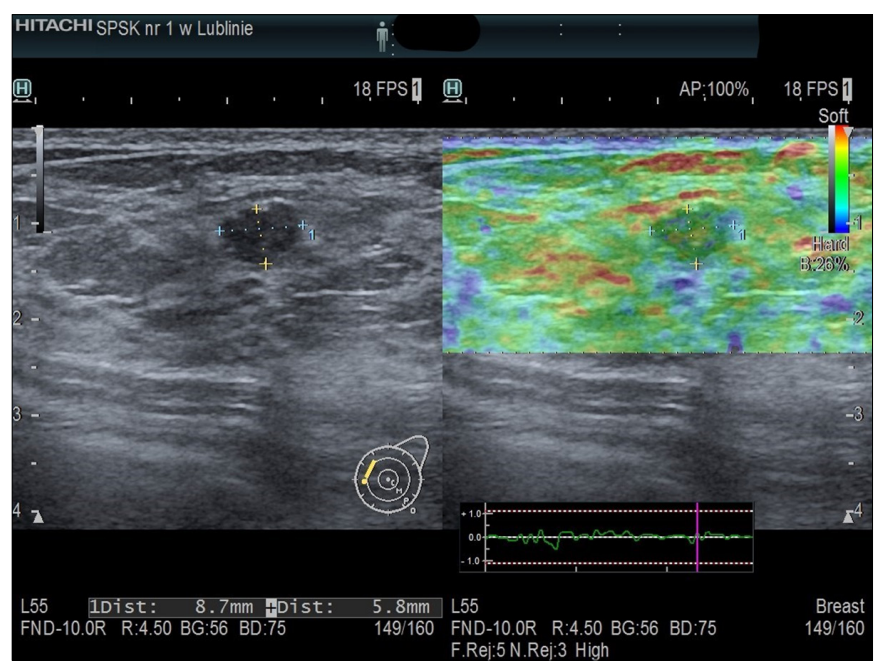

FIGURE 2. Hypoechoic lesion with the dimensions 9x6 $\mathrm{mm}$ classified as Tsukuba score 2.

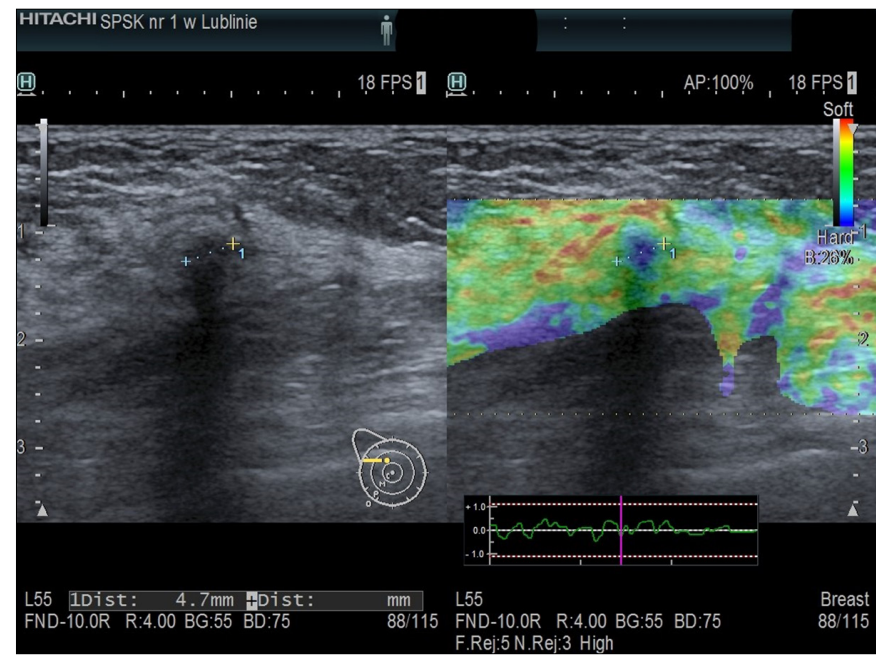

FIGURE 3. Hypoechoic lesion with the dimensions $5 \times 5 \mathrm{~mm}$ classified as Tsukuba score 3.

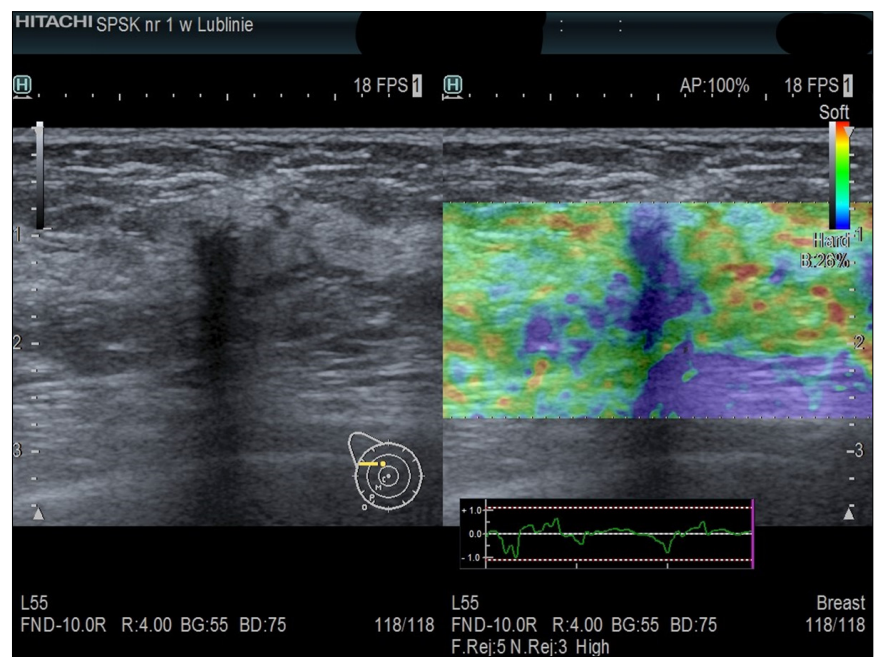

FIGURE 4. Hypoechoic lesion classified as Tsukuba score 4.

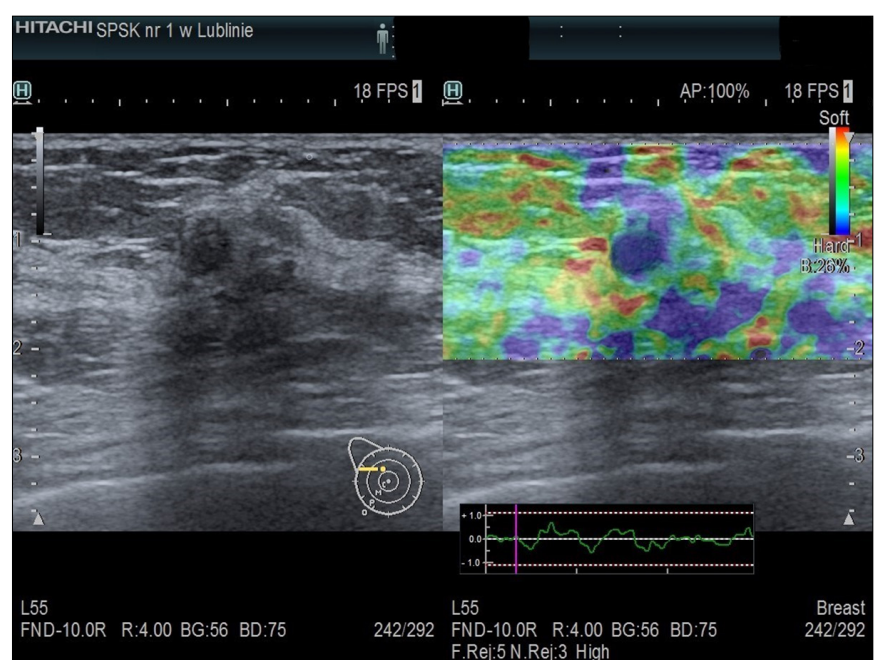

FIGURE 5. Hypoechoic lesion classified as Tsukuba score 4.

The sensitivity and specificity of the Tsukuba scale are the greatest for the cut-off point between benign and malignant lesion, which corresponds to a grade of $3 / 4$ of the scale [12]. Another type of compressive elastography, used in clinical practice, is the examination using the tissue Doppler imaging method (TDI). The tissue Doppler imaging method is based on measuring of shear velocity of tissue under pressure and relaxation [10]. In turn, dynamic elastography is based on tissue deformation under the acoustic radiation force. The basic 
parameter of the elastic properties of the tissues is the Young's modulus, which is a physical, objective measurement of the stiffness [13].

The studies have shown that USE significantly improves the interpretation of non-invasive examinations such as MMG and ultrasound. The purpose of the study by Mohey N. et al. was to evaluate the value of USE and B-mode ultrasound in the differentiating of benign and malignant solid breast tissue changes detected in MMG. A number of 100 patients with a total of 114 lesions detected in MMG were enrolled in the study. Tissue changes discovered during MMG were categorized into benign or malignant and further verified in USE. The diagnostic results of MMG and USE were compared with histopathological findings. On the basis of histopathological results, 33 out of 114 lesions were malignant, and 81 were benign. Ultrasound elastography, compared to MMG and USG, was the most specific modality (95.1\%). The accuracy of USE $(81.7 \%)$ was comparable to MMG $(82.5 \%)$ and was higher than in ultrasound $(71.9 \%)$. Furthermore, a combination of USE and B-mode ultrasound had the best sensitivity (90.9\%) and detection accuracy $(93.8 \%)$ in the differentiation of solid changes in the breast [14].

Another study by Fischer T, et al. assessed the possibility of improving the differentiation of focal breast tissue changes using USE. Based on the evaluation of 201 women with a total of 85 benign and 116 malignant lesions it has been shown that USE with quantitative SR calculation (SRs) is characterized by high sensitivity (95\%) and specificity (74\%). These values were significantly higher compared to the classic B-mode ultrasound (sensitivity and specificity $85 \%$ and $60 \%$, respectively) and MMG (78\% and 62\%) [15].

The aim of the next study was to evaluate the value of USE compared to the ultrasound B-mode examination in the differentiation of focal breast tissue changes and to determine the usefulness diagnostic value of the BIRADS-ultrasound classification and Tsukuba scale. The examined group included 80 women with 99 solid focal lesions in the breasts (39 malignant lesions and 60 benign ones). Based on the study, it has been shown that combined application of both examinations conventional ultrasound and USE, helps in the assessment of solid breast tissue changes. In addition, the use of both scales, BIRADS-ultrasound and Tsukuba, contributes to the improvement of the sensitivity $(87.2 \%)$ and specificity $(95 \%)$ of character differentiation of focal lesions. In the case of problematic tissue changes classified as BIRADS-ultrasound grade 3 , the verification in USE showed grades 1 and 2 in Tsukuba scale and confirmed their benign nature [16].

Another important role of USE is estimation of early response to chemotherapy in patients with locally advanced breast cancer. Study by Katyan et al. (2019) was performed to evaluate role of USE in rating the response to neoadjuvant chemotherapy (NACT) in patients with locally advanced breast cancer (LABC). The number of 86 women with LABC, receiving NACT, were included to the study. USE was performed before each cycle of chemotherapy and before surgery by two independent researchers.

In the USE the changes in elastographic parameters, such as size ratio and deformation ratio, were evaluated. The results were compared to clinical and pathologic tumor response assessed after mastectomy. The USE showed high sensitivity (97.7\%) and moderate specificity (68.7\%) in determining the response to the first cycle of NACT. In contrast, USE sensitivity and specificity parameters for estimating responses to the second and third cycles of NACT were satisfactory. Based on this study, it was shown that USE is a reliable and earliest predictor of treatment response in patients with LABC. Moreover, systematic examinations during chemotherapy may help in evaluation the degree of response to preoperative treatment, which may help in therapeutic decisions in patients with breast cancer [17]. However, it should be noted that USE is not a perfect method. The limitation in its application is mainly the size of the lesions. The study by Choi (2019) et al. compared the performance of B-mode ultrasound, USE and the combination of B-mode ultrasound and USE in breast lesions $\leq 2 \mathrm{~cm}$. Women with a total of 428 small focal breast lesions, of which 142 were histopathologically verified as malignant and 268 were benign, were included. The study showed that the addition of USE to B-mode ultrasound improve sensitivity and specificity in the differentiation of breast tissue changes. However, in the case of small changes and carcinoma in situ a tendency to false negative results has been shown. In turn, factors such as the patient's older age, short distance to the skin or chest wall correlated with false positive results. Despite the diagnostic utility of USE in small breast lesions $(\leq 2 \mathrm{~cm})$, the lesion size, pathology type and location were associated with false results [18].

\section{CONCLUSIONS}

Focal breast tissue changes have various elastic properties, which allows the use of elastography to suspect their character. The data presented in this review provide information on advantages and disadvantages of USE in determining the nature of the lesions. It should be emphasized that USE plays an important role in verification tissue changes assessed in the B-mode ultrasound examination as BIRADS 3. Conventional B-mode ultrasound is characterized by high sensitivity, but lower specificity. The addition USE to ultrasound B-mode in common clinical practice improves differentiation the character of focal lesions in the breasts.

\section{REFERENCES}

1. Didkowska J, Wojciechowska U. Nowotwory złośliwe w Polsce w 2013 roku. Warszawa: Centrum Onkologii - Instytut im. M. SkłodowskiejCurie; 2015

2. Oeffinger KC, Fontham ET, Etzioni R, et al. Breast cancer screening for women at average risk: 2015 Guideline Update From the American Cancer Society. JAMA. 2015;314(15):1599-614.

3. D’Orsi CJ, Sickles EA, Mendelson EB, Morris EA. ACR BI-RADS ${ }^{\circledR}$ Atlas, Breast Imaging Reporting and Data System. Reston, VA. American College of Radiology; 2018.

4. Carney PA, Miglioretti DL, Yank Askas BC, et al. Individual and combined effects of age, breast density, and hormone replacement the rapyuse on the accuracy of screening mammography. Ann Intern Med. 2003;138:168-75.

5. Costantini M, Belli P, Lombardi R, et al. Characterization of solid Breast Masses. J Ultrasound Med. 2006;25:649-61.

6. Gennisson JL, Deffieux T, Fink M, Tanter M. Ultrasound elastography: principles and techniques. Diagn Interv Imaging. 2013;94:487-95.

7. Sigrist RMS, Liau J, Kaffas AE, et al. Ultrasound elastography: Review of techniques and clinical applications. Theranostics. 2017;7(5):1303-29.

8. Faruk T, Islam MK, Arefin S, Haq MZ. The Journey of Elastography: Background, Current Status, and Future Possibilities in Breast Cancer Diagnosis. Clin Breast Cancer. 2015;15(5):313-24.

9. Barr RG. Sonographic breast elastography: a primer. J Ultrasound Med 2012;31:773-83.

10. Nowicki A, Dobruch-Sobczak K. Introduction to ultrasound elastography. J Ultrason. 2016;16(65):113-24. 
11. Itoh A, Ueno E, Tohno E, et al. Breastdisease: clinicalapplication of US elastography for diagnosis. Radiology. 2006;239:341-50.

12. Cho N, Jang M, Lyou CY, et al. Distinguishing benign from malignant masses at breast US: combined US elastography and color Doppler US-influence on radiologist accuracy. Radiology. 2012;262:80-90.

13. Ophir J, Céspedes I, Ponnekanti H, et al. Elastography: aquantitative method for imaging the elasticity in biologicaltissues. Ultrason Imaging. 1991;13:111-34.

14. Mohey N, Hassan TA. Value of mammography and combined grey scale ultrasound and ultrasound elastography in the differentiation of solid breast lesions. Egypt J Radiol Nucl Med. 2014;45:253-61.

15. Fischer T, Peisker U, Fiedor S, et al. Significant differentiation of focal breast lesions: raw data-based calculation of strain ratio. Ultraschall in der Medizin. 2012;33:372-9.

16. Dobruch-Sobczak K. The differentiation of the character of solid lesions in the breast in the compression sonoelastography. Part II: Diagnostic value of BIRADS-US classification, Tsukuba score and FLR ratio. J Ultrason. 2013;13(52):31-49.

17. Katyan A, Mittal MK, Mani C, Mandal AK. Strain wave elastography in response assessment to neo-adjuvant chemotherapy in patients with locally advanced breast cancer. Br J Radiol. 2019;92(1099):20180515.

18. Choi HY, Seo M, Sohn YM, et al. Shear wave elastography for the diagnosis of small $(\leq 2 \mathrm{~cm})$ breast lesions: added value and factors associated with false results. Br J Radiol. 2019;92(1097):20180341.
Corresponding author

Łukasz Kwietniewski

Department of Radiology, Medical University of Lublin,

16 Staszica St., 20-081Lublin

tel: +48815321084

E-mail: lukaszkwietniewski85@gmail.com 